

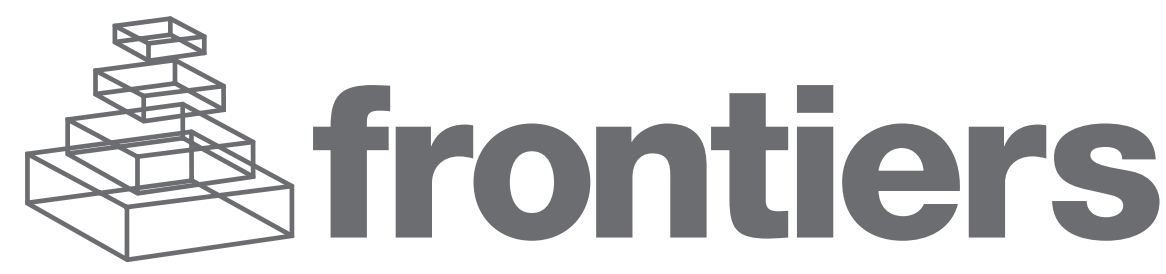

\section{FRONTIERS COPYRIGHT} STATEMENT

C Copyright 2007-2015

Frontiers Media SA

All rights reserved.

All content included on this site, such as text, graphics, logos, button icons, images, video/audio clips, downloads, data compilations and software, is the property of or is licensed to Frontiers Media SA

("Frontiers") or its licensees and/or subcontractors. The copyright in the text of individual articles is the property of their respective authors, subject to a license granted to Frontiers.

The compilation of articles constituting this e-book, wherever published, as well as the compilation of all other content on this site, is the exclusive property of Frontiers. For the conditions for downloading and copying of e-books from Frontiers' website, please see the Terms for Website Use. If purchasing Frontiers e-books from other websites or sources, the conditions of the website concerned apply.

Images and graphics not forming part of user-contributed materials may not be downloaded or copied without permission.

Individual articles may be downloaded and reproduced in accordance with the principles of the CC-BY licence subject to any copyright or other notices. They may not be re-sold as an e-book.

As author or other contributor you grant a CC-BY licence to others to reproduce your articles, including any graphics and third-party materials supplied by you, in accordance with the Conditions for Website Use and subject to any copyright notices which you include in connection with your articles and materials.

All copyright, and all rights therein, are protected by national and international copyright laws.

The above represents a summary only. For the full conditions see the Conditions for Authors and the Conditions for Website Use.

\section{ABOUT FRONTIERS}

Frontiers is more than just an open-access publisher of scholarly articles: it is a pioneering approach to the world of academia, radically improving the way scholarly research is managed. The grand vision of Frontiers is a world where all people have an equal opportunity to seek, share and generate knowledge. Frontiers provides immediate and permanent online open access to all its publications, but this alone is not enough to realize our grand goals.

\section{FRONTIERS JOURNAL SERIES}

The Frontiers Journal Series is a multi-tier and interdisciplinary set of open-access, online journals, promising a paradigm shift from the current review, selection and dissemination processes in academic publishing.

All Frontiers journals are driven by researchers for researchers; therefore, they constitute a service to the scholarly community. At the same time, the Frontiers Journal Series operates on a revolutionary invention, the tiered publishing system, initially addressing specific communities of scholars, and gradually climbing up to broader public understanding, thus serving the interests of the lay society, too.

\section{DEDICATIONTO QUALITY}

Each Frontiers article is a landmark of the highest quality, thanks to genuinely collaborative interactions between authors and review editors, who include some of the world's best academicians. Research must be certified by peers before entering a stream of knowledge that may eventually reach the public - and shape society; therefore, Frontiers only applies the most rigorous and unbiased reviews.

Frontiers revolutionizes research publishing by freely delivering the most outstanding research, evaluated with no bias from both the academic and social point of view.

By applying the most advanced information technologies, Frontiers is catapulting scholarly publishing into a new generation.

\section{WHAT ARE FRONTIERS RESEARCHTOPICS?}

Frontiers Research Topics are very popular trademarks of the Frontiers Journals Series: they are collections of at least ten articles, all centered on a particular subject. With their unique mix of varied contributions from Original Research to Review Articles, Frontiers Research Topics unify the most influential researchers, the latest key findings and historical advances in a hot research area!

Find out more on how to host your own Frontiers Research Topic or contribute to one as an author by contacting the Frontiers Editorial Office: researchtopics@frontiersin.org 


\section{THE ROLE OF PHYSICAL FITNESS ON CARDIOVASCULAR RESPONSES TO STRESS}

Topic Editors:

Daniel A. Boullosa, Universidade Católica de Brasília, Brazil

Arto J. Hautala, Verve Research, Finland

Anthony S. Leicht, James Cook University, Australia

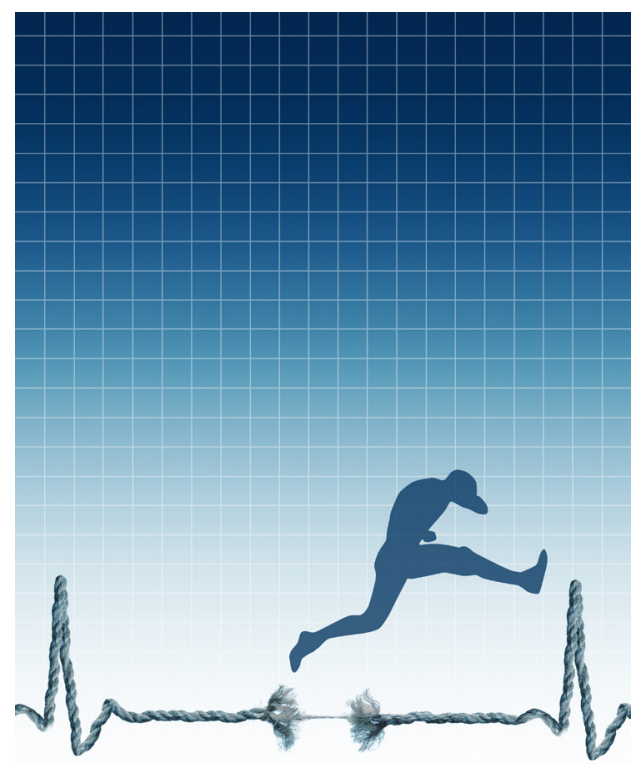

A greater fitness can help individuals to jump the hurdle of stress.

Image created by Pilar Boullosa.
Cardiovascular responses to physical and/ or mental stressors has been a topic of great interest for some time. For example, significant changes of cardiovascular control and reactivity have been highlighted as important mechanisms for the protective effect of exercise as a simple and effective, non medical therapy for many pathologies. However, despite the great number of studies performed to date (e.g. $>54,000$ entries in Pubmed for "cardiovascular stress"), important questions of the role stress has on cardiovascular function still remain. For instance, What factors account for the different cardiovascular responses between mental and physical stressors? How do these different components of the cardiovascular system interact during stress? Which cardiovascular responses to stress are the most important for identifying normal, depressed, and enhanced cardiovascular function? Can these stress-induced responses assist with patient diagnosis and prognosis? What impact does physical fitness have on the relationship between cardiovascular function and health? The current topic examined our current understanding of cardiovascular responses to stress and the significant role that physical fitness has on these responses for improved function and health. Manuscripts focusing on heart rate variability (HRV), heart rate recovery, and other novel cardiovascular assessments were especially encouraged. 


\section{Table of Contents}

04 Introduction to the Research Topic: The Role of Physical Fitness on Cardiovascular Responses to Stress

Daniel A. Boullosa, Arto J. Hautala and Anthony S. Leicht

06 Cardiovascular Reactivity, Stress, and Physical Activity

Chun-Jung Huang, Heather E. Webb, Michael C. Zourdos and Edmund O. Acevedo

19 Vagal Modulation of Resting Heart Rate in Rats: The Role of Stress,

Psychosocial Factors, and Physical Exercise

Luca Carnevali and Andrea Sgoifo

31 The Role of Physical Activity and Heart Rate Variability for the Control of Work Related Stress

Laís Tonello, Fabio B. Rodrigues, Jeniffer W. S. Souza, Camen S. G. Campbell, Anthony S. Leicht and Daniel A. Boullosa

40 The Interplay Between Stress and Physical Activity in the Prevention and Treatment of Cardiovascular Disease

Matthew A. Stults-Kolehmainen

44 Mindfulness May Both Moderate and Mediate the Effect of Physical Fitness on Cardiovascular Responses to Stress: A Speculative Hypothesis

Marcelo M. P. Demarzo, Jesús Montero-Marin, Phyllis K. Stein, Ausiàs Cebolla, Jaime G. Provinciale and Javier García-Campayo

52 Regular Exercise is Associated with Emotional Resilience to Acute Stress in Healthy Adults

Emma Childs and Harriet de Wit

59 The Impact of Escitalopram on Vagally Mediated Cardiovascular Function to Stress and the Moderating Effects of Vigorous Physical Activity: A Randomized Controlled Treatment Study in Healthy Participants

Camilla S. Hanson, Tim Outhred, Andre R. Brunoni, Gin S. Malhi and Andrew H. Kemp

70 Traditional Games Resulted in Post-Exercise Hypotension and a Lower Cardiovascular Response to the Cold Pressor Test in Healthy Children Suliane B. Rauber, Daniel A. Boullosa, Ferdinando O. Carvalho, José F. V. N. de Moraes, lorranny R. C. de Sousa, Herbert G. Simões and Carmen S. G. Campbell

77 Reduced Vasodilator Function Following Acute Resistance Exercise in Obese Women

Nina C. Franklin, Mohamed Ali, Melissa Goslawski, Edward Wang and Shane A. Phillips

84 Central Gene Expression Changes Associated with Enhanced Neuroendocrine and Autonomic Response Habituation to Repeated Noise Stress After Voluntary Wheel Running in Rats

Sarah K. Sasse, Tara J. Nyhuis, Cher V. Masini, Heidi E. W. Day and Serge Campeau 


\title{
Introduction to the research topic: the role of physical fitness on cardiovascular responses to stress
}

\author{
Daniel A. Boullosa ${ }^{1 *}$, Arto J. Hautala ${ }^{2}$ and Anthony S. Leicht ${ }^{3}$ \\ 1 Post-Graduate Program in Physical Education, Catholic University of Brasilia, Águas Claras, Brazil \\ ${ }^{2}$ Department of Exercise and Medical Physiology, Verve Research, Oulu, Finland \\ ${ }^{3}$ College of Healthcare Sciences, James Cook University, Townsville, QLD, Australia \\ *Correspondence: d_boullosa@yahoo.es
}

Edited by:

Mikko Paavo Tulppo, Verve, Finland

Reviewed by:

Harri Lindholm, Finnish Institute of Occupational Health, Finland

Keywords: physical activity, stress, exercise, cardiovascular diseases, physical fitness

This e-book is the culmination of countless hours of meticulous work by global scientists. We would like to thank the researchers for their great contributions to this hot topic. The combination of these studies reflects the importance of the topic amongst researchers and practitioners and the wide interest from numerous laboratories around the world. The contributions include a variety of formats including five original investigations, three review articles, one opinion article and a hypothesis and theory article. Notably, these contributions included both human and animal models that encompassed a range of techniques from molecular mechanisms to real life interventions thus reinforcing the translational approach for the understanding of cardiovascular responses to stress.

The three review articles (Huang et al., 2013; Carnevali and Sgoifo, 2014; Tonello et al., 2014) provided a great insight into the current knowledge of cardiovascular stress and its relationship with physical activity (PA). The first review article by Huang et al. (2013) considered the big picture of the topic by combining the classical perspective-examining how different forms of induced cardiovascular stress can be attenuated in physically trained individuals, with the addition of the interrelationships between obesity, inflammation and oxidative stress with these stress responses and cardiovascular health. The subsequent review from Carnevali and Sgoifo (2014) involved animal studies that focused on a mechanistic approach with resting vagal tone leading to stress resilience, reducing the development of anxiety and depression, and the important role of PA to mediate these relationships. The mini-review from Tonello et al. (2014) extended the topic into one of the most important social stressors in modern life, the work environment. This review revealed that factors related to adverse working conditions such as excessive effort, effort-reward imbalance, over commitment, irregular shift work, and work stress were associated with reduced cardiac autonomic function. Importantly this review identified the need for further studies in this area with heart rate variability (HRV) recognized as an important tool for evaluating both work related stress including harmful physical inactivity, and adaptations to potentially important stress buffers as PA and enhanced physical fitness.
Following the review articles, two interesting papers addressed novel and important aspects for stress management therapies. The opinion article by Stults-Kolehmainen (2013) highlighted an important and frequently forgotten aspect: How does stress negatively influence PA levels in individuals with and without cardiovascular diseases? Additionally, Stults-Kolehmainen (2013) introduced the concept of "mindfulness" for reducing stress and subsequently enhancing patients PA levels. Subsequently, Demarzo et al. (2014) discussed potentially effective means to mediate the role of physical fitness on cardiovascular responses to stress using "mindfulness" interventions that may be applied to this new area of future research.

As mentioned previously, the variety of original research investigations included within this topic reinforced the importance of research translation. The cross-sectional study by Childs and de Wit (2014) reported a significant relationship between positive affect decline after a social stressor and regular exercise in healthy individuals despite no difference between exercisers and non-exercisers for post-stress heart rate, blood pressure and cortisol responses. This study effectively illustrated how regular PA enhances psychological health and strengthens emotional resilience to stressors. Likewise, the study of Hanson et al. (2013) provided additional support for the effectiveness of regular PA in attenuating physiological responses to social stressors. Interestingly, an antidepressant drug attenuated cardiovascular responses (i.e., HR and HRV) to stress only in irregular exercisers that exhibited a comparable stress-induced response similar to that of regular exercisers during placebo (Hanson et al., 2013). These two studies (Hanson et al., 2013; Childs and de Wit, 2014) highlighted the positive impact that regular PA has on mental health, especially for depressed patients, with further studies needed to elaborate on mechanisms, benefits, and potential new therapies.

Other important responses during different sources of stress were also included in this e-book. The study of Rauber et al. (2014) was the first to our knowledge that documented postexercise hypotension (PEH) in children. This study (Rauber et al., 2014) reported an enhanced PEH after traditional games compared to active video game playing and watching TV, 
and an attenuated blood pressure response during the cold pressor test following traditional games. The greater exercise intensity and metabolic demands of traditional games were highlighted as important factors for these responses with children's playing strategies fundamental for cardiovascular health.

The study of Franklin et al. (2014) documented the important contribution of other physiological responses during stress. These authors (Franklin et al., 2014) noted that endothelial function after resistance exercise (i.e., physical stressor) was impaired for obese women compared to lean women with endothelium independent-vasodilation correlated to body weight for all women. These findings provided important guidance for resistance exercise prescription of obese women to minimize cardiovascular disease risk.

Finally, the study of Sasse et al. (2013) presented a very novel hypothesis suggesting that exercise might facilitate adaptation to repeated stress via both hypothalamic-pituitary-adrenocortical axis and cardiovascular response habituation. In this study (Sasse et al., 2013), the brains of rats that performed voluntary exercise on a wheel and those that were sedentary were analyzed following control, acute and repeated noise exposures. These authors identified that unlike sedentary rats, exercising rats regulated corticotropin-releasing factor and brain derived neurotrophic factors across several brain regions. Subsequently, the hypothesis was supported with habituation to stress using exercise resulting in multiple system responses. Future studies may elaborate on the results of Sasse et al. (2013) to understand the response of different physiological systems and their interactions to stressors and applicable translation.

This e-book has taken the initial action to integrate current research findings to stimulate further discussion and research. We would like to thank the authors for their significant contributions and the many reviewers who critiqued and improved the overall topic. Future studies will clarify the importance of physical fitness, exercise and PA to regulate cardiovascular responses during stress and such benefits for cardiovascular health.

\section{REFERENCES}

Carnevali, L., and Sgoifo, A. (2014). Vagal modulation of resting heart rate in rats: the role of stress, psychosocial factors, and physical exercise. Front. Physiol. 5:118. doi: 10.3389/fphys.2014.00118
Childs, E., and de Wit, H. (2014). Regular exercise is associated with emotional resilience to acute stress in healthy adults. Front. Physiol. 5:161. doi: 10.3389/fphys.2014.00161

Demarzo, M. M. P., Montero-Marin, J., Stein, P. K., Cebolla, A., Provinciale, J. G., and García-Campayo, J. (2014). Mindfulness may both moderate and mediate the effect of physical fitness on cardiovascular responses to stress: a speculative hypothesis. Front. Physiol. 5:105. doi: 10.3389/fphys.2014.00105

Franklin, N. C., Ali, M., Goslawski, M., Wang, E., and Phillips, S. A. (2014). Reduced vasodilator function following acute resistance exercise in obese women. Front. Physiol. 5:253. doi: 10.3389/fphys.2014.00253

Hanson, C. S., Outhred, T., Brunoni, A. R., Malhi, G. S., and Kemp, A. H. (2013). The impact of escitalopram on vagally mediated cardiovascular function to stress and the moderating effects of vigorous physical activity: a randomized controlled treatment study in healthy participants. Front. Physiol. 4:259. doi: 10.3389/fphys.2013.00259

Huang, C.-J., Webb, H. E., Zourdos, M. C., and Acevedo, E. O. (2013). Cardiovascular reactivity, stress, and physical activity. Front. Physiol. 4:314. doi: 10.3389/fphys.2013.00314

Rauber, S. B., Boullosa, D. A., Carvalho, F. O., de Moraes, J. F. V. N., de Sousa, I. R. C., Simões, H. G., et al. (2014). Traditional games resulted in post-exercise hypotension and a lower cardiovascular response to the cold pressor test in healthy children. Front. Physiol. 5:235. doi: 10.3389/fphys.2014.00235

Sasse, S. K., Nyhuis, T. J., Masini, C. V., Day, H. E. W., and Campeau, S. (2013). Central gene expression changes associated with enhanced neuroendocrine and autonomic response habituation to repeated noise stress after voluntary wheel running in rats. Front. Physiol. 4:341. doi: 10.3389/fphys.2013.00341

Stults-Kolehmainen, M. A. (2013). The interplay between stress and physical activity in the prevention and treatment of cardiovascular disease. Front. Physiol. 4:346. doi: 10.3389/fphys.2013.00346

Tonello, L., Rodrigues, F. B., Souza, J. W. S., Campbell, C. S. G., Leicht, A. S., and Boullosa, D. A. (2014). The role of physical activity and heart rate variability for the control of work related stress. Front. Physiol. 5:67. doi: 10.3389/fphys.2014.00067

Conflict of Interest Statement: The authors declare that the research was conducted in the absence of any commercial or financial relationships that could be construed as a potential conflict of interest.

Received: 03 September 2014; accepted: 03 November 2014; published online: 19 November 2014.

Citation: Boullosa DA, Hautala AJ and Leicht AS (2014) Introduction to the research topic: the role of physical fitness on cardiovascular responses to stress. Front. Physiol. 5:450. doi: 10.3389/fphys.2014.00450

This article was submitted to Clinical and Translational Physiology, a section of the journal Frontiers in Physiology.

Copyright (C) 2014 Boullosa, Hautala and Leicht. This is an open-access article distributed under the terms of the Creative Commons Attribution License (CC BY). The use, distribution or reproduction in other forums is permitted, provided the original author(s) or licensor are credited and that the original publication in this journal is cited, in accordance with accepted academic practice. No use, distribution or reproduction is permitted which does not comply with these terms. 